\title{
Application of 3D Electromagnetic Articulography in Dentistry: Mastication and Deglutition Analysis. Protocol Report
}

\author{
Aplicación de la Articulografía Electromagnética 3D en Odontología: \\ Análisis de la Masticación y Deglución. Reporte de Protocolo
}

\author{
Ramón Fuentes 1,2; Fernando Dias 1,2; Giannina Álvarez ${ }^{3}$; María Florencia Lezcano ${ }^{2,4}$; \\ Constanza Farfán ${ }^{2,3}$; Nicolás Astete ${ }^{3}$ \& Alain Arias Al, $^{1,5}$
}

FUENTES, R.; DIAS, F.; ÁLVAREZ, G.; LEZCANO, M. F.; FARFÁN, C.; ASTETE, N. \& ARIAS, A. Application of 3D electromagnetic articulography in dentistry: mastication and deglutition analysis. Protocol report. Int. J. Odontostomat., 12(1):105-112, 2018.

ABSTRACT: The Stomatognathic System (SS) is an integrated and coordinated morphofunctional unit, consisting of skeletal, muscular, angiological, nervous, glandular and dental structures that work in harmony to perform different functions such as chewing, swallowing, speaking, breathing, tasting and esthetics. The study of SS physiology is crucial to achieving a correct maintenance of its normal function or restoration. Thus, we developed a protocol to record mandibular movement, chewing and swallowing (saliva and water) by using the 3D-electromagnetic articulograph AG501 at the Oral Physiology Laboratory of the Research Centre for Dental Sciences (CICO). This tool has been used for decades in speech and phonetic research and it is able to record, present and evaluate articulated structure movement data in real time. The articulograph provides an ordered data matrix (values for the $x, y, z$ axes, among others) for the recorded movement, which is stored in binary files that can be processed by different software. One of these is MATLAB ${ }^{\circledR}$, allowing us to perform a quick, clear and understandable analysis. The existence of a standardized methodology for recording allows an accurate description of the stages to perform different types of measurements according to the researcher's objective. This has opened the way for a new type of oral physiology analysis based on study reproducibility, which is considered one of the bases of scientific methodology. swallowing.

KEY WORDS: electromagnetic articulography; AG501; stomatognathic system; odontology, mastication,

\section{INTRODUCTION}

The Stomatognathic System (SS) is an integrated and coordinated morphofunctional unit, consisting of a set of skeletal, muscular, angiological, nervous, glandular and dental structures (Barreto, 1999). These structures work in harmony to perform different functions such as chewing, swallowing, speaking, breathing, tasting and esthetics (Manns, 2013). The importance of studying the physiology of these structures must be stressed in order to achieve a correct maintenance of SS normal function or restoration (Mizraji et al., 2012).
The masticatory function is characterized by motor, rhythmic and learned responses, which are combined with reflection and voluntary motions, with conscious control from start to finish (Manns, 2011); chewing can be intentionally accelerated, slowed or stopped (Watanabe et al., 2015). Many factors in SS can affect masticatory function; however, the most affected is dental state (Hatch et al., 2001; Lepley et al., 2011). The description of more specific patterns of chewing movements is very necessary, since this would contribute to the accurate diagnosis of oral functions

\footnotetext{
${ }^{1}$ Department of Integral Adult Dentistry, Dental School, Universidad de La Frontera, Temuco, Chile.

${ }^{2}$ Research Centre for Dental Sciences (CICO), Dental School, Universidad de La Frontera, Temuco, Chile.

${ }^{3}$ Master's Program in Dentistry, Dental School, Universidad de La Frontera, Temuco, Chile.

${ }^{4}$ Engineering School, Universidad Nacional de Entre Ríos, Entre Ríos, Argentina.

${ }^{5}$ Universidad Adventista de Chile, Chillán, Chile.
} 
(Fuentes et al., 2015). Evaluation of the chewing process can be done by analyzing the number of chewing cycles, cycle length and their respective phases (time), mandibular displacement and mean mandibular speed (Karlsson \& Jemt, 1991). Other studies have complemented these analyses by adding variables such as the maximum speed of the phases of each chewing cycle and mean mandibular opening (Gonçalves et al., 2014).

Furthermore, swallowing involves reflex sequences that transport food from the oral cavity to the stomach, while protecting the respiratory tract at the same time (Felton et al., 2007). It is considered a voluntary, involuntary and reflex activity (Okeson, 2008), in which neuromuscular coordination, central nervous system involvement, sensorial stimulations and motor responses of voluntary and involuntary muscles are necessary (Arvedson \& Brodsky, 2002). It consists of four phases, with the first two being voluntary and the last two reflexes; dysfunction in any of these phases can alter swallowing. Regarding deglutition, studies mainly focus on describing different swallowing patterns (Williamson et al., 1990; Yagi et al., 2015), tongue movement in the sagittal plane (Bourdiol et al., 2014) and intraoral pressure behavior during this process (Kydd \& Toda, 1962; Hind et al., 2005; Kennedy et al., 2010).

Among all the available methodologies for recording, describing and analyzing SS functions, Electromagnetic Articulography (EMA) stands out; it has been used in speech and phonetic research for decades (Ozbek et al., 2003; Katz \& McNeil, 2010; Narayanan et al., 2014). EMA was recently certified by the U.S. Federal Communications Commission as a low-power communication device that uses electromagnetic fields with a frequency of 7.5 to 13.75 $\mathrm{KHz}$ (Fuentes et al., 2017). Its use is mainly highlighted in the study of tongue movements during pronunciation and swallowing (Chen et al., 2007) and in the analysis of mandibular movements, with high spatial and temporal resolution capacity (Fuentes et al., 2017). Therefore, in this report we describe the development of a technique and protocol to record mandibular, chewing and swallowing movements (tongue movement) in volunteer participants. Electromagnetic articulography was used for this purpose, as it provides a comprehensive, intuitive, comprehensible and fast analysis when data are processed with programming software capable of generating measurement calculations and other variables, along with graphs in different spatial planes for their interpretation.

\section{METHOD DESCRIPTION}

Electromagnetic Articulography. EMA systems can record, present and assess articulated structure movement in real time. Among EMA systems on the market, we find $A G$ articulographs developed by Carstens Medizinelektronik (Bovenden, Germany). Their latest model, the AG501 3D articulograph (Fig. 1A), is an improved version with less error rate than its predecessor, the AG500 articulograph. The AG501 3D articulograph has 9 transmitter coils, with each generating alternate electromagnetic fields at different frequencies. It also has 16 receiver coils or sensors that must be placed on the participant and on the articulatory organs under study. Due to the influence of electromagnetic fields, a small alternating electric current is induced in the sensors, whose parameters are recorded by the articulograph. The value of the induced electric current parameters (voltage, amplitude) is a function of the distance between the sensor and the respective transmitter coil, whereby variation in the values is used to determine the spatial position of the active sensors in time. This is possible through complex mathematical modeling, which allows us to record the spatial position of each sensor, using values in the three spatial axes $(x, y, z)$ contained in an ordered data matrix (Fig. 1B). This data matrix also contains values for two rotational angles (f, q) and one pair of extra values ("rms" - root median square). The amount of positional data for each of the sensors that the articulograph can record over time, depends on the sampling frequency. Thus, the AG501 3D articulograph can record at a frequency ranging from 250 to $1250 \mathrm{~Hz}$ (or data per second). All this information, arranged in an ordered data matrix, is stored and exported as a digital binary file (.pos), which can be subsequently processed by different software.

In order to ensure an accurate recording of the sensor positions, it is necessary to verify that the participant's articulatory organ (for example, the head) and the sensors placed therein are within the spherical measurement area when recording (Fig. 1A). The measurement area is a representation of the electromagnetic field, generated by the transmitter coils of the articulograph obtained through complex mathematical modeling. The representation of this spherical field has a diameter of $300 \mathrm{~mm}$. It is also necessary to establish a reference system to standardize positional records of sensors placed on specific articulatory organs (such as the jaw or tongue), and which belong to a larger moving system or structure (such as the head). Thus, the recording of sensor movement 


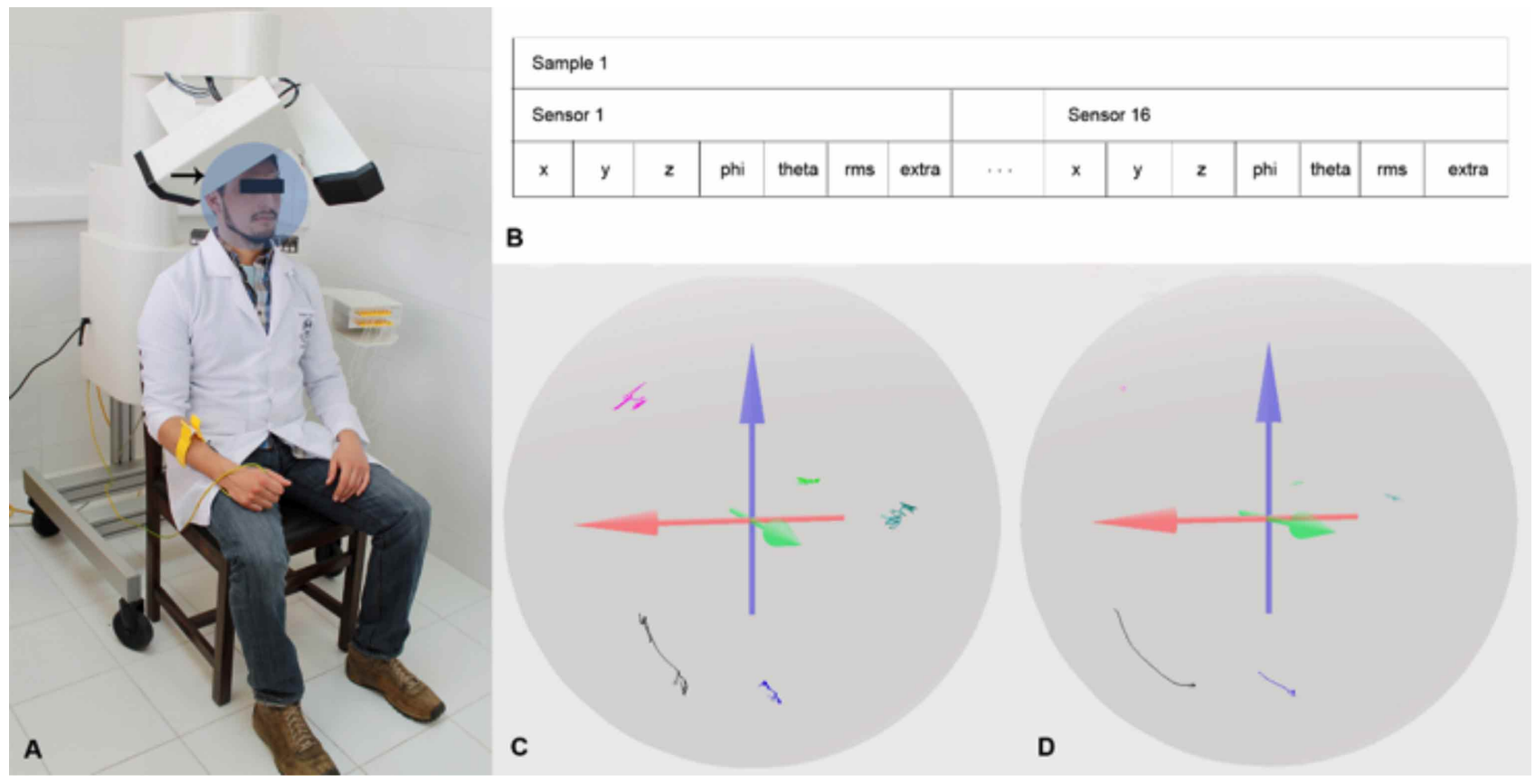

Fig. 1. A) AG5013D electromagnetic articulograph (Carstens Medizinelektronik, Bovenden, Germany) from the Oral Physiology Laboratory of the Research Center for Dental Sciences (CICO), Universidad de La Frontera. The articulograph has 9 transmitter coils and 16 receiver coils or sensors that can be placed on the participant or subject of study. (Arrow) Field of spherical measurement of $300 \mathrm{~mm}$ diameter of the articulograph. The participant's head with the placed sensors must be within this field of measurement to ensure the accuracy of the recordings. B) Matrix scheme of ordered data provided by the articulograph. The output format of this data matrix is a binary file (.pos), which can be processed by different software. C) Standardization scheme of recorded movement and establishment of a reference system. Absolute movement in which the whole system moves and the absolute position (movement) of each sensor is recorded. B) Relative movement, in which a reference system of three sensors (violet, green and light green points) is established that is used to present the movement of the rest of active sensors (black and blue lines). Although the entire system (sensor set) moves, the distance between the reference sensors and the active sensor does not change, so the movement is not relative, but absolute.

placed on the jaw or the tongue, is relative to a previously established reference system that allows us to set aside the absolute movement of the entire system (Fig. 1C, D). In order to achieve this, Head Correction function is used according to the instructions for use described in the manufacturer's manual and in Fuentes et al. (2017).

Positional data processing. The digital binary files exported by the articulograph are processed and analyzed in order to obtain geometric (trajectory, distance, area) and kinematic parameters (time, speed) of the recorded movement. It is possible to use different software for the processing of these files, and our research group developed reports with two: VisArtico and MATLAB®. While VisArtico (Ouni et al., 2012) allows a direct visualization of the movement or route of the sensor recorded in the files, obtaining positional values (for the $x, y, z$ axis) at any point of the sensor's route (Fig. 2). MATLAB® (The MathWorks, Inc., Natick, MA,

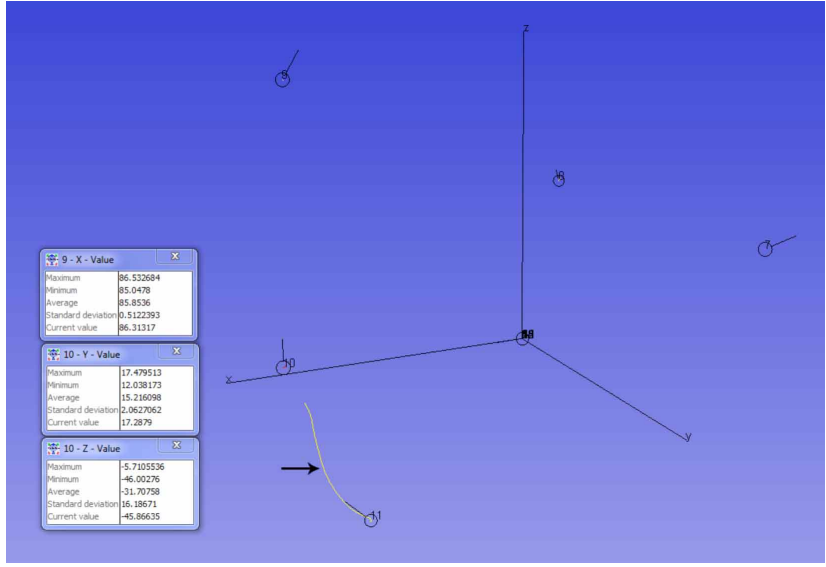

Fig. 2. Visualization in VisArtico software of the sensor movements recorded by the AG5013D electromagnetic articulograph. In this case, mandibular opening movement (arrow). With this software, it is possible to obtain the positional data of any point in the movement trajectory (boxes). 
USA), allows a quick, clear and understandable analysis through the creation of specific calculation routines or scripts (Fuentes et al., 2017). The scripts developed by our research group are based on vector calculations and allow us to calculate the trajectories, distances, areas (geometric characteristics), time and speed (kinematic characteristics) of the recorded movement, based on the positional values stored in the ordered data matrix. Moreover, these movements can be visualized with this software, by generating graphs in time, or at a given instant. In order to achieve these specific calculations, we must create files (.pos) with the matrix of sensor positional data when performing each individual movement. By recording each movement separately, it is possible to analyze their characteristics in the different spatial planes and achieve a comprehensive analysis.

Protocol for recording jaw and chewing movement. Our research group developed a protocol to record and subsequently analyze jaw and chewing movement through the use of the AG5013D electromagnetic articulograph and MATLAB®. In order to do this, five sensors were arranged at the following points of the participant's head: The skin point corresponding to the

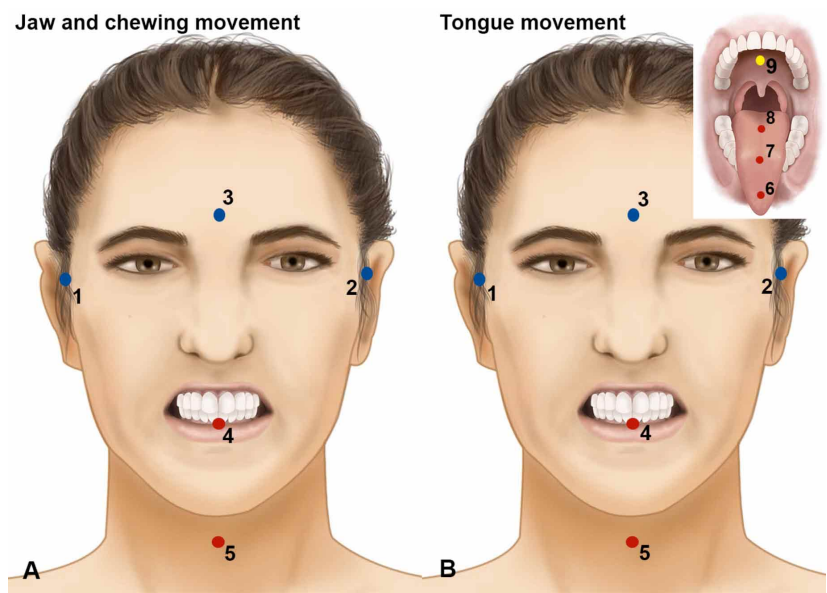

Fig. 3. A) For the protocol for recording jaw and chewing movement. Distribution of the articulograph sensors placed at specific points on the patient's head: skin point of the right (1) and left (2) mastoid (behind ears), glabella (3), mid-incisive mandibular midline (4) and skin point of the thyroid cartilage (5). B) For the recording protocol of tongue movement when swallowing saliva and water: distribution of the articulograph sensors placed at specific points on the patient's head. Skin point of the right (1) and left (2) mastoid, glabella (3), mandibular mid-incisive midpoint (4), skin point of the thyroid cartilage (5), anterior (6), middle (7) and posterior (8) portion of the tongue. An extra sensor (9) was used to define the sagittal profile of the hard and soft palate. right (1) and left (2) mastoid, glabella (3), mandibular inter-incisor midline (4) and the skin point of the thyroid cartilage (5) (Fig. 3A). The first three sensors (skin point of the right and left mastoid and glabella) were used as a reference system to standardize the recording of the sensors that were located on the jaw and skin point of the thyroid cartilage (Head Correction function). The sensor placed at the skin point of the thyroid cartilage was used to detect the end of the pharyngeal phase of swallowing, once the participant had been asked to chew a test food.

In order to begin recording with the articulograph, the participants (and the fixed sensors) were placed in a chair, under the articulograph transmitter coils, and told to adopt a comfortable and erect position while looking straight ahead. Then, each participant was asked to perform a series of mandibular movements, prior instruction by the operator, in order to describe Posselt's envelope in the frontal and sagittal plane. The movements were recorded independently and in order, according to the description in Table I. Once the mandibular movements were recorded, the participant was asked to chew $3 \mathrm{~g}$ of peanuts and $5 \mathrm{~g}$ of carrots, independently. Therefore, the chewing cycles were recorded for each type of food until the participant swallowed. For each type of recording (jaw and chewing movement), different types of graphs and calculations were generated for the three anatomical axes (x-craniosacral, y-lateral-lateral, z-dorsoventral), using MATLAB® (Fig. 4).

Protocol for recording tongue movement in swallowing. As in the previous case, this protocol was developed using the AG5013D electromagnetic articulograph and MATLAB $®$. Thus, it was possible to record tongue movement during the swallowing of saliva (spontaneously and at rest) and water (using different volumes). In this case, eight sensors were used that were placed on specific points on the participant. These points were: skin point corresponding to the right (1) and left (2) mastoid, glabella (3), mandibular inter-incisive midline (4), skin point of the thyroid cartilage (5), anterior (6), middle (7) and posterior (8) portion of the tongue (Fig. 3B). As in the previous protocol, the first three sensors (skin point of the right and left mastoid and glabella) were used to execute the Head Correction function. The sensor placed on the mandibular inter-incisive midline was used to establish the beginning of swallowing through a visual observation of the mouth opening, while the sensor placed at the cutaneous point of the thyroid cartilage, was used to detect the end of the 
FUENTES, R.; DIAS, F.; ÁLVAREZ, G.; LEZCANO, M. F.; FARFÁN, C.; ASTETE, N. \& ARIAS, A. Application of 3D electromagnetic articulography in dentistry: mastication and deglutition analysis. Protocol report. Int. J. Odontostomat., 12(1):105-112, 2018.
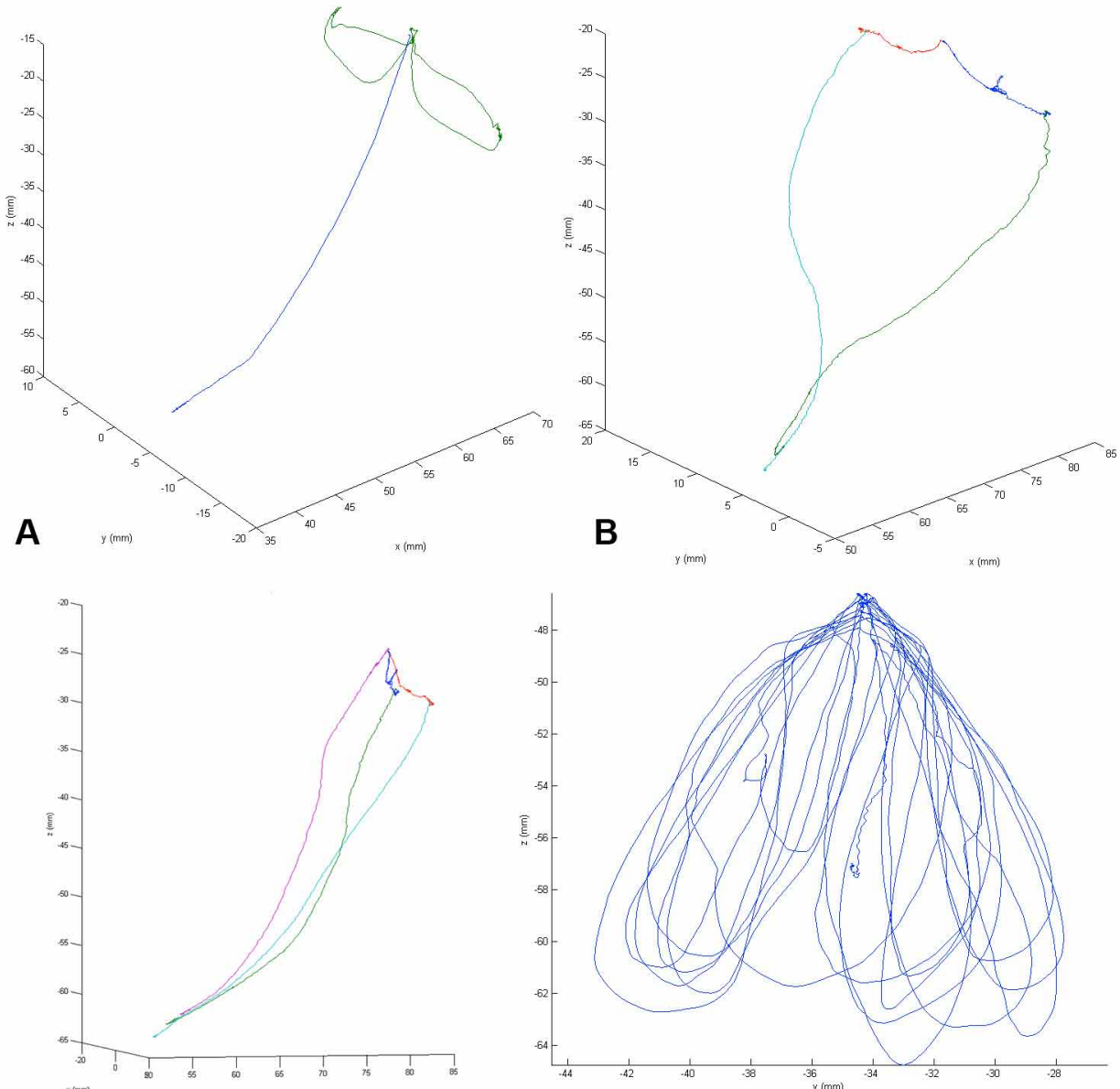

$C^{x(m m)}$

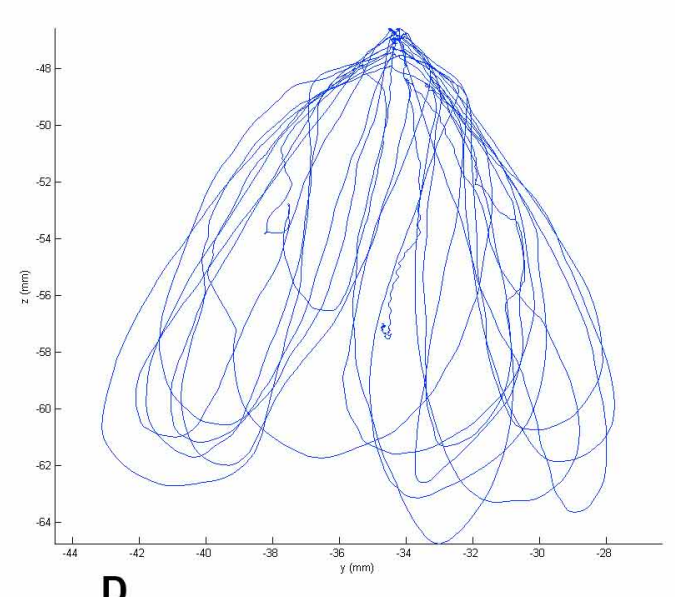

Fig. 4. Graphs generated in MATLAB $®$ (in 3D) of the movement recorded with the AG501 $3 \mathrm{D}$ electromagnetic articulograph. A) Opening and laterality movement without any right or left contact. B) Envelope of contacting and bordering jaw movements in the frontal plane. C) Envelope of contacting and bordering jaw movements in the sagittal plane. D) Chewing cycles.

Table I. Mandibular movements for the description of Posselt's diagram in the frontal and sagittal plane (mentioned in order according to recording protocol). All the movements begin from the position of maximum intercuspation.

\begin{tabular}{cll}
\hline $\mathbf{n}^{\mathbf{0}}$ & Mandibular Movement & \\
\hline 1 & Maximum opening & Maximum non-contacting laterality (to the right and left, passing through \\
2 & mandibular postural position) & Posselt's diagram in the \\
3 & Maximum contact right laterality & frontal plane \\
4 & Maximum bordering opening right & Posselt's diagram in the \\
5 & Maximum contact left laterality & sagittal plane \\
6 & Maximum bordering opening left & \\
7 & Edge-to-edge & \\
9 & Maximum contact protrusion & Maximum opening in protrusion \\
11 & Maximum contact retrusion &
\end{tabular}


swallowing pharyngeal phase. The three sensors placed on the tongue were on the midline: one on the anterior portion at $12 \mathrm{~mm}$ from the tip of the tongue, the second on the posterior part of the lingual dorsum that is tolerated by the participant and the third on the middle of the tongue, equidistant from the anterior and posterior sensors. An extra sensor (9) was used to define the sagittal profile of the hard and soft palate, which was attached to a wooden rod covering the entire palate through the midline from the base of the uvula to the inter-incisive papilla. Recording the palate profile is useful to relate tongue movement during swallowing with palate height.
In order to record tongue movement during spontaneous swallowing (at rest) and when swallowing water (using different volumes), participants (and the fixed sensors) were positioned in a chair, under the articulator transmitter coils, and told to adopt a comfortable and erect position, while looking straight ahead.

Swallowing saliva at rest. After executing the Head Correction function, the participant was instructed to closely watch a 10-minute video, with the only indication of staying focused, relaxed, with his or her mouth closed and lips together. Thus, recordings of 1-min duration
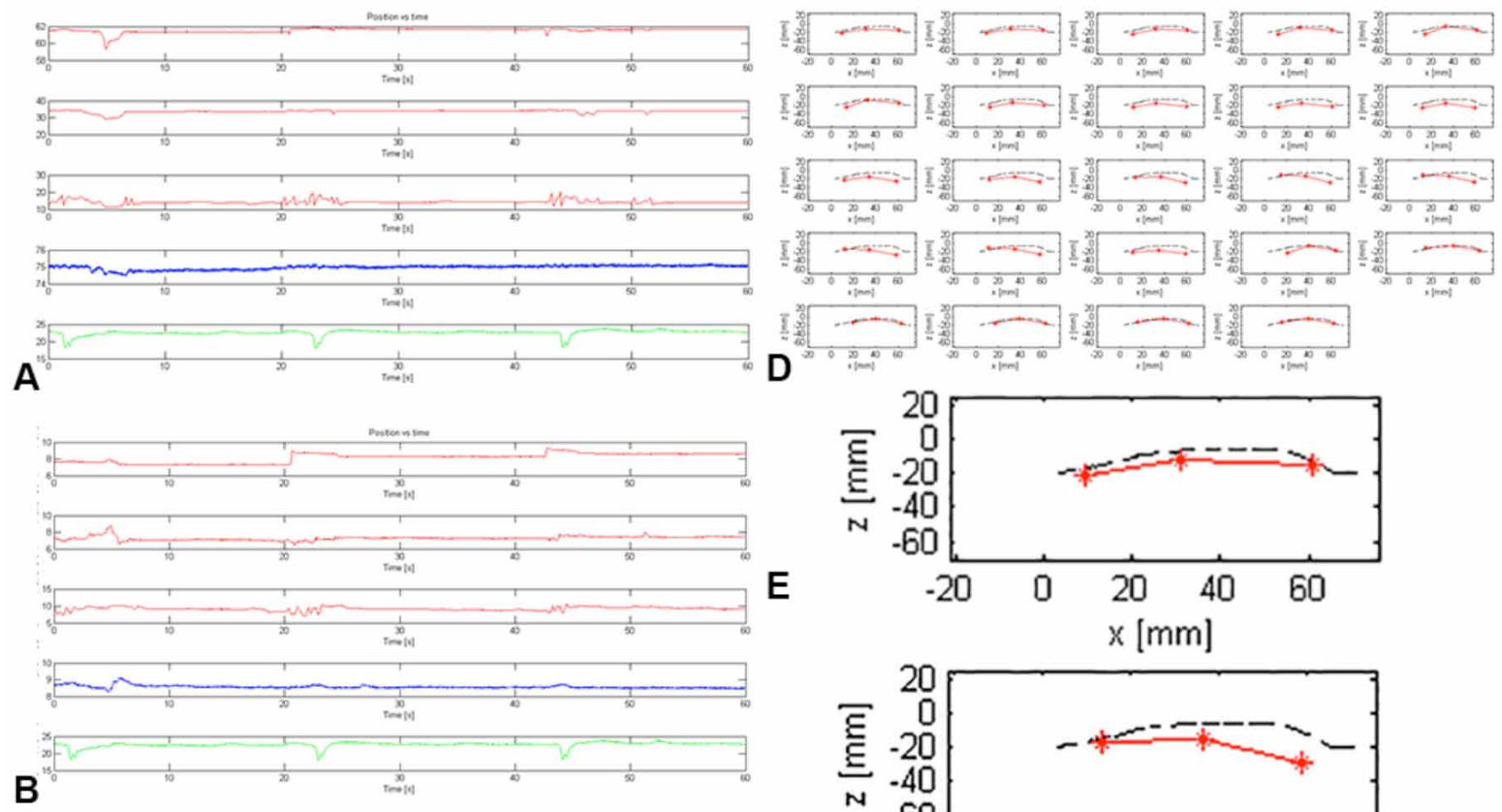

E
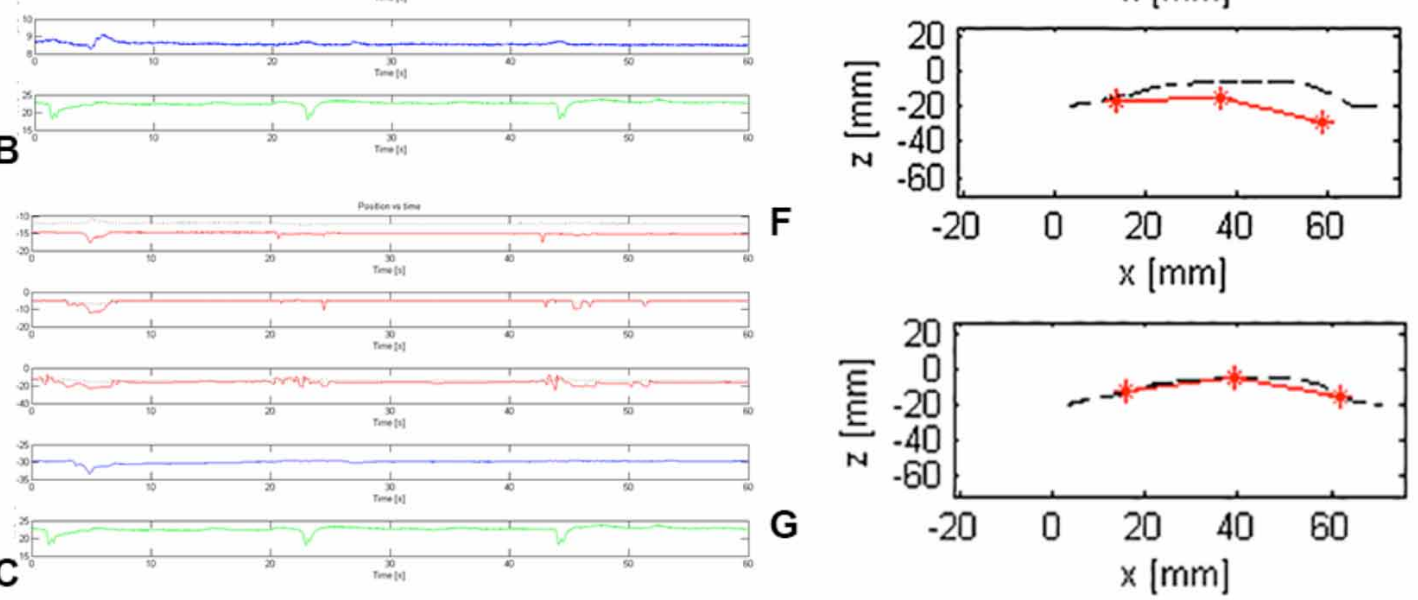

Fig. 5. Graphs generated in MATLAB® of the movement recorded with the AG501 3D electromagnetic articulograph. Time graphs of sensor movement on the tongue (anterior, middle and posterior; red lines), jaw (blue line) and skin point of the thyroid cartilage (green line) in the three anatomical axes: $x$-craniosacral (A) ylateral-lateral (B) and z-dorsoventral (C). Special types of Fig.s composed by a sequence of articulated segment graphs (D) were generated, representing a specific time at the beginning $(E)$, middle $(F)$ and final of swallowing (G). These articulated segments are formed by the union of three points (corresponding to the three sensors placed on the tongue: anterior, middle and posterior) through straight lines. 
were performed in which the spontaneous swallowing of saliva was expected to be observed. The video was used to divert the participant's attention from the recording process and to avoid external (environmental) or internal influences (awareness of sensors and cables in mouth, pressure or stress, etc.) that may alter the swallowing process. For this recording, through MATLAB ${ }^{\circledR}$, time graphs of sensor movement on the tongue (anterior, middle and posterior), jaw and skin point of the thyroid cartilage were generated in the three anatomical axes (x-craniosacral, $y$-lateral-lateral, z-dorsoventral), correlating the height of the hard and soft palate in the latter (Fig. 5A, B, C). In addition, special types of figures composed by a sequence of articulated segment graphs were generated (Fig. $5 \mathrm{C}$ to $\mathrm{G}$ ). These articulated segments are formed by the union of three points (corresponding to the three sensors placed on the tongue: anterior, middle and posterior) through straight lines.

Swallowing water. In order to record tongue movement while swallowing water, an average volume per swallow (AVS) was established for each participant. AVS is a fluid volume that varies in each subject and allows a comfortable, easy and one-time swallow. To determine this, the participant was asked to take a sip of water five times from a millimeter glass. Each time the participant drank water, the volume of swallowed water was recorded, and an average or AVS was obtained. Thus, after executing the Head Correction function, the patient was instructed to swallow 1AVS, 12 AVS, V4 AVS, and 2 AVS in separate recordings, and this was done three times for each. For this recording, through MATLAB, time graphs of the sensor movements on the tongue (anterior, middle and posterior), jaw and skin point of the thyroid cartilage were generated in the three spatial planes, as well as figures composed by a sequence of articulated segment graphs (Fig. 5).

\section{CONCLUSIONS}

The movement records performed by the AG5013D electromagnetic articulograph were stored as positional data, which can be processed with software such as MATLAB ${ }^{\circledR}$ and VisArtico. The development of specific calculation routines in MATLAB $®$ allowed us to perform a complete threedimensional analysis of the movement characteristics related to certain SS functions. Additionally, the movement assessment through VisArtico provided us with multimedia input (videos) for a more intuitive and simple analysis. Through both analyses, it was possible to study the movement with a high degree of accuracy, whose margin of error is negligible due to the functional characteristics and articulograph configuration. This entire process was also successful due to the standardized recording methodology, which we described in detail for each stage, in order to achieve different types of measurements (such as trajectories, distances, areas, speed) according to the researcher's objective. Thus, the door is opened to a new form of analysis in oral physiology that is based on study reproducibility, which is considered one of the bases of scientific methodology.

\section{ACKNOWLEDGMENTS}

Ramón Fuentes and his research group have received support from the Research Office, Universidad de La Frontera. This work is part of the project DI16-0049 approved by the Universidad de La Frontera Scientific Ethics Committee (approval number 038/ 2016).

FUENTES, R.; DIAS, F.; ÁLVAREZ, G.; LEZCANO, M. F.; FARFÁN, C.; ASTETE, N. \& ARIAS, A. Aplicación de la articulografía electromagnética en odontología: análisis de la masticación y deglución. Reporte de protocolo. Int. J. Odontostomat., 12(1):105-112, 2018.

RESUMEN: EI Sistema Estomatognático es una unidad morfo-funcional integrada y coordinada, constituida por estructuras esqueléticas, musculares, angiológicas, nerviosas, glandulares y dentales que actúan en armonía para realizar diferentes funciones como la masticación, deglución, fonoarticulación, respiración, degustación y estética. Es importante el estudio de la fisiología del SE para lograr un correcto mantenimiento de su función normal, o para la restauración de este. Es así como en el Laboratorio de Fisiología Oral del Centro de Investigación en Ciencias Odontológicas (CICO) hemos desarrollado un protocolo para el registro del movimiento mandibular, masticación y deglución (saliva y agua), utilizando el articulógrafo electromagnético 3D AG501, el cual ha sido utilizado desde hace décadas en la investigación del habla y la fonética y que es capaz de grabar, presentar y evaluar en tiempo real datos del movimiento de estructuras articuladas. El articulógrafo provee de una matriz de datos ordenados (valores para los ejes $x, y, z$, entre otros) para el movimiento registrado, el cual se almacena en archivos binarios que pueden procesarse a través de diferentes softwares. Uno de ellos es MATLAB ${ }^{\circledR}$, el cual nos permite realizar un análisis de forma rápida, clara y comprensible. La existencia de una estandarización 
metodológica de registro, permite describir de forma exacta las etapas para llevar a cabo distintos tipos de mediciones según el objetivo del investigador. De esta forma se abre la puerta a una nueva forma de análisis en la fisiología oral que se sustenta en la reproducibilidad de la investigación que es considerada una de las bases de la metodología científica.

PALABRAS CLAVE: articulografía electromagnética; AG501; sistema estomatognático; odontología; masticación; deglución.

\section{REFERENCES}

Arvedson, J. \& Brodsky, L. Pediatric Swallowing and Feeding, Assessment and Management. $2^{\text {nd }}$ ed. San Diego, Singular Thomson Learning, 2002.

Barreto, J. F. Sistema estomatognático y esquema corporal. Colomb. Med., 30(4):173-80, 1999.

Bourdiol, P.; Mishellany-Dutour, A.; Peyron, M. A. \& Woda, A. Tonguemandible coupling movements during saliva swallowing. J. Oral. Rehabil., 41(3):199-205, 2014.

Chen, H. Y.; Murdoch, B. E.; Goozée, J. V. \& Scott, D. Physiologic development of tongue-jaw coordination from childhood to adulthood. J. Speech Lang. Hear. Res., 50(2):352-60, 2007.

Felton, S. M.; Gaige, T. A.; Reese, T. G.; Wedeen, V. J. \& Gilbert, R. $\mathrm{J}$. Mechanical basis for lingual deformation during the propulsive phase of swallowing as determined by phase-contrast magnetic resonance imaging. J. Appl. Physiol. (1985), 103(1):255-65, 2007.

Fuentes, R.; Arias, A.; Saravia, D.; Lezcano, M. F. \& Dias, F. J. An innovative method to analyse the range of border mandibular movements using 3D electromagnetic articulography (AG501) and MATLAB. Biomed. Res. (India), 28(9):4239-47, 2017.

Fuentes, R.; Navarro, P.; Curiqueo, A. \& Ottone, N. E. Determination of mandibular border and functional movement protocols using an electromagnetic articulograph (EMA). Int. J. Clin. Exp. Med., 8(11):19905-16, 2015.

Gonçalves, T. M.; Vilanova, L. S.; Gonçalves, L. M. \& RodriguesGarcia, R. C. Effect of complete and partial removable dentures on chewing movements. J. Oral Rehabil., 41(3):177-83, 2014.

Hatch, J. P.; Shinkai, R. S.; Sakai, S.; Rugh, J. D. \& Paunovich, E. $D$. Determinants of masticatory performance in dentate adults. Arch. Oral Biol., 46(7):641-8, 2001.

Hind, J. A.; Nicosia, M. A.; Gangnon, R. \& Robbins, J. A. The effects of intraoral pressure sensors on normal young and old swallowing patterns. Dysphagia, 20(4):249-53, 2005.

Karlsson, S. \& Jemt, T. Adaptive changes of masticatory movement characteristics after rehabilitation with osseointegrated fixed prostheses in the edentulous jaw: a 10-year follow-up study. Int. J. Oral. Maxillofac. Implants, 6(3):259-63, 1991.

Katz, W. F. \& McNeil, M. R. Studies of articulatory feedback treatment for apraxia of speech based on electromagnetic articulography. Perspect. Neurophysiol. Neurogenic Speech Lang. Disord., 20:73-79, 2010.

Kennedy, D.; Kieser, J.; Bolter, C.; Swain, M.; Singh, B. \& Waddell, J. N. Tongue pressure patterns during water swallowing. Dysphagia, 25(1):11-9, 2010.

Kydd, W. L. \& Toda, J. M. Tongue pressures exerted on the hard palate during swallowing. J. Am. Dent. Assoc., 65(3):319-30, 1962.

Lepley, C. R.; Throckmorton, G. S.; Ceen, R. F. \& Buschang, P. H.
Relative contributions of occlusion, maximum bite force, and chewing cycle kinematics to masticatory performance. Am. J. Orthod. Dentofacial Orthop., 139(5):606-13, 2011.

Manns, A. Sistema Estomatognático. Fundamentos Clínicos de Fisiología y Patología Funcional. Santiago de Chile, Amolca, 2013.

Manns, A.; Biotti, J.; Brizuela, C.; Dolwick, M.; Fresno, M. \& González, H. Sistema Estomatognático: Fisiología y su Correlaciones Clínicas-Biológicas. Madrid, Médica Ripano, 2011.

Mizraji, M.; Manns Freese, A. \& Bianchi, R. Stomatognathic system. Actas Odontol., 9(2):35-47, 2012.

Narayanan, S.; Toutios, A.; Ramanarayanan, V.; Lammert, A.; Kim, J.; Lee, S.; Nayak, K.; Kim, Y. C.; Zhu, Y.; Goldstein, L.; Byrd, D.; Bresch, E.; Ghosh, P.; Katsamanis, A. \& Proctor, M. Real-time magnetic resonance imaging and electromagnetic articulography database for speech production research (TC). J. Acoust. Soc. Am., 136(3):1307, 2014.

Okeson, J. P.; Sosa, G.; Bumann, A.; Bumann, U. \& Lotzmann, U. Tratamiento de Oclusión y Afecciones Temporomandibulares. $5^{\text {th }}$ ed. Amsterdam, Elsevier, 2008.

Ouni, S.; Mangeonjean, L. \& Steiner, I. VisArtico: A Visualization Tool for Articulatory Data. Interspeech 2012, September 9-13, Portland, 2012.

Ozbek, M.; Tulunoglu, I.; Ozkan, S. \& Oktemer, M. Evaluation of articulation of Turkish phonemes after removable partial denture application. Braz. Dent. J., 14(2):125-31, 2003.

Watanabe, Y.; Hirano, H. \& Matsushita, K. How masticatory function and periodontal disease relate to senile dementia. Jpn. Dent. Sci. Rev., 51(1):34-40, 2015.

Williamson, E. H.; Hall, J. T. \& Zwemer, J. D. Swallowing patterns in human subjects with and without temporomandibular dysfunction. Am. J. Orthod. Dentofacial Orthop., 98(6):507-11, 1990.

Yagi, K.; Lowe, A. A.; Ayas, N. T.; Fleetham, J. A. \& Almeida, F. R. Swallowing and breathing patterns during sleep in patients with obstructive sleep apnea. Sleep Breath, 19(1):377-84, 2015.

Corresponding author:

Prof. Dr. Ramón Fuentes Fernández

Research Centre for Dental Sciences

Dental School, Universidad de La Frontera

Av. Francisco Salazar 01145

Temuco

CHILE

Email: ramon.fuentes@ufrontera.cl

Received:11-10-2017

Accepted: 14-12-2017 